\title{
Discussion on the Importance of Design Literacy to the Development of Fine Arts Education in Middle Schools
}

\author{
${ }^{1}$ JiMei University, Jimei, Xiamen, Fujian, China \\ 2JiMei University, Jimei, Xiamen, Fujian, China \\ 1140751800@qq.com \\ b*635235972@qq.com
}

Qiduan Chen ${ }^{1, \text { a }}$,Shichen $\operatorname{Lin}^{2,}{ }^{*}$

\begin{abstract}
As the reform on art curriculum standard for compulsory education continues in China, the proportion of such curriculum in elementary and secondary schools comprised of design education increases rapidly. As the key to design education, the concept of design literacy has attracted experts' and scholars' attention in the past ten years. However, there has been little research done on art education in secondary schools regarding the connotation of design literacy and the importance of teaching the skill. This research will review and discuss relevant documents about design literacy from China and other countries, then explore the concept of design literacy based on current grounded theory, while analyzing the importance of design literacy on art education in secondary schools. Research has found that design literacy plays an active role in cultivating middle school students' basic abilities, enhancing critical thinking skills, and strengthening positive emotional attachments to the subject. Insights gained from this research will assist fine arts teachers to revise the current environments for art education or will offer guidelines and references for educators to establish meaningful secondary school art education curriculum in the future.
\end{abstract}

Keywords: Design Education, Design Research, Component Discussion, Documentary Analysis

\section{设计素养对中学美术教育发展之重要性探讨}

陈其端 ${ }^{1, a}$ ， 林诗琛 ${ }^{2,}$ b*

${ }^{1}$ 集美大学，集美，厦门，福建，中国

2集美大学, 集美, 厦门, 福建, 中国

a1140751800@qq.com

b*635235972@qq.com

\section{摘要}

随着目前国内义务教育美术课程标准改革的不断深入,设计教育课程在中小学美术教育课程中的比重也在逐渐 上升，而设计素养作为设计教育中的关键，近十年来备受广大专家学者的关注。就中学美术教育而言，探讨设 计素养意涵及强调设计素养在教育中重要性的研究相对较少。本研究将回顾设计素养相关之国内外文献, 以设 计、艺术、教育三大领域作为分类, 进行扎根理论并探讨设计素养之意涵, 分析设计素养在中学美术教育中的 重要性。研究发现设计素养对培养中学生基本能力、关键能力、思维品质与情感态度具有积极作用, 研究成果 可协助目前美术教师进行现行教学环境检视或为未来中学美术教育课程建构提供参考作用。

关键词: 设计教育, 设计研究, 成分探讨, 文献分析 


\section{1.前言}

20 世纪 20 年代, 西方设计教育发展迅速, 我国 设计教育起步虽晚, 但自改革开放后也逐渐进入蓬勃 发展的时期。近 20 年教育界将设计逐步融入到中小 学美术教育, 使其更好地渗透到大众的观念与学习 中，但设计却始终无法如同阅读、计算一般成为每个 人必备的基本素养。导致这个问题出现的原因与许多 人不了解设计素养甚至忽视设计素养在教育中的作 用有关, 故本研究旨在通过探讨设计素养之意涵及分 析设计素养在中学美术教育中的作用, 以此强调设计 素养的重要性从而促进中学美术教育的发展与大众 设计素养的提升。

\section{2.设计教育中的设计素养}

在每一个平凡的日常、每一个饱含意义的细节、 或是每一种追求更美好的生活态度中, 我们因为微不 足道却新鲜的事物发出“哇”的一声感叹, 这种感觉就 像开始有点抗拒, 但很快就会像对待礼物一样欣然接 受。如今对我们来说, 设计就是这样的存在。而“设 计师”这个称号, 也终于脱离了传统职业的桎梏, 属 于我们平凡日常中的每一个人。随着时代发展, 为了 使设计更好地得到普及并提升大众能力, 设计教育便 产生了。

放眼国际, 德国于 1919 年成立世界第一所为发 展设计而建的学院——包豪斯设计学院, 其“艺术与 技术统一”的现代设计艺术教育理念对世界的设计教 育影响至深。英国的设计教育则是伴随着工业革命的 进行不断发展起来的, 1837 年, 英国成立了皇家艺 术学院 (RCA), 旨在以工业设计引导工业生产适应 消费市场的需求, 后经历了一百多年的发展, 设计教 育随着 1988 年《教育改革法》的颁布, 在英国中小 学的课程中发挥其重大的作用。聚焦国内, 以美术学 科为例, 我国曾于 2001 年颁发试行的《美术新课程 标准 (实验稿) 》义务教育阶段的“设计·应用”和高中 教育阶段的“设计.工艺”强调对学生创造能力和动手 能力的培养。[1]随着现代设计在我国蓬勃发展, 教育 中重美术、轻设计的观念也通过美术课程标准的改革 不断改变着。

谈及设计教育, 一定离不开对设计素养的研究。 Liv Merete Nielsen 与 Karen Braenne(2013)在提到欧盟 教育体系时强调, 设计教育的实施不应采取自上而下 的方法, 应从长远的角度与教育相联系。其中联结的 关键正是一个不为大众所熟知的词汇一一设计素养。
[2]2012 年，欧洲设计领导委员会向欧盟委员会提出了 21 项关于欧洲未来发展的建议, 其中第 20 项建议提 出: “通过在教育系统的每个层次培养一种“人人学习 设计”的文化, 提高欧洲所有公民的设计素养水平。” 但设计素养的培养不是一蹴而就的, 它需要长期的酝 酿与磨炼, 这就要求在中小学阶段普及设计教育, 使 学生有足够的时间形成正确的设计态度与观念, 从而 提高学生的设计素养。

\section{3.设计与素养概念界说}

素养，顾名思义指一个人平日的修养。这个词汇 在教育环境中并不陌生, 如今它也被越来越多地使用 在教学中, 并出现了科学素养、文学素养、数学素养、 批判性素养等组合词汇, 设计素养也正是融合了设计 与素养的概念。若想分析设计素养之意涵, 首先需要 了解素养与设计的概念。

强汾生 (2000) 曾提出: 素养是指一个人的修养, 它包括道德素养、外在气质、知识水平与能力等各个 方面, 这个定义较为广泛, 并为大众所熟知。 ${ }^{[3]}$ 后来 张定强（2019）在这个基础上提出自己的见解, 他认 为素养是指一个人的品德、知识、才能和体格等在先 天的条件和后天的学习与锻炼中形成的综合结果, 通 常理解为先赋性条件与后致性因素相互作用并通过 训练和实践而获得的思维品格与能力。 ${ }^{[4]}$ 在国内, 素 养的意涵往往离不开人的修养品德与知识技能, 但国 外看待素养的角度却不同, 联合国教科文组织于 2004 年对素养一词进行定义, 提出素养是指能够理解、解 释、创造、交流和计算的能力, 并且需要连续地学习, 使个人能够实现他们的目标, 发展他们的知识和潜 力。这就说明, 提升素养并非一朝一夕, 所以中小学 教育对学生素养的培养十分重要。

就设计的定义而言, 著名学者李砚祖认为设计是 人类改变原有事物, 使其变化、增益、更新、发展的 创造性活动。在《汉语大词典（1993 年版）》中, 对设计的定义是“根据一定要求, 对某项工作预先制 定图样、方案。”[5]可以说, 设计伴随着人类的发展, 人类演进史中随处可见设计的影子, 如今更是存在于 我们日常生活的每个角落。

\section{4.设计素养意涵探讨}

设计素养融合了设计与素养的概念, 但在国内对 于设计素养的定义至今尚无统一的定论, 即使在国 外, 由于各个国家设计教育的实施方法不同, 故对设 计素养的定义各有侧重又存在一定的交叉。笔者对国 
内外相关文献按年序进行整理，以艺术、设计、教育 三大领域作为分类,进行扎根理论并探讨三大领域中 设计素养的重要影响因子, 以此归纳分析设计素养之 意涵, 如表 1 。

\section{1. 设计领域之设计素养因子}

欲探讨设计素养之意涵, 首先需要对设计领域进 行探索。我们的衣食住行无不与设计有关, 但由于设 计领域的广泛性，使得其分类很难界定。从设计的目 的性来说, 设计主要是根据不同功能和人的需求, 确 立物质生产领域的功能显示与审美形式, 以生产出符 合人的使用需要和审美价值的产品。按照设计与人和 自然的关系以及目前社会的生活环境与条件, 我们可 以将设计领域划分为视觉传达设计、产品设计、环境 设计三大领域, 并在这些领域相关文献里找寻设计素 养的重要影响因子。

薛拥军 (2009) 通过教学实践总结了室内设计专 业学生特点, 旨在提升室内设计专业学生的设计素 养。他提出设计素养是一种设计能力、设计创新能力、 设计职业素养以及社会责任感的综合能力表现, 其中 设计能力包括擅长形象思维、逻辑思维能力与专业知 识基础; 设计创新能力要求学生具备创新思维的同时 坚持原创; 设计职业素养则包括严谨的纪律观念、对 设计工作具有一定的紧迫感、责任感、一定的团队协 作能力以及人际交往能力; 而社会责任感则培养学生 对他人对社会的道德情感。[6]薛拥军将知识、能力与 社会情感整合为设计素养, 符合大众对“素养”的一般 认知, 但设计素养的意涵仅限于此吗?

冯荣 (2014) 的研究对设计素养意涵进行了扩充, 她提出在文化创意产业中设计素养的首要评价标准 为社会责任感, 该观点与薛拥军一致, 不过冯荣更强 调对待设计工作的热情以及设计要以人为本, 为大众 所服务。此外冯䒯还提出文化底蕴和团队精神也是设 计素养不可或缺的部分, 设计作品的永恒性取决于其 中的文化内涵, 且良好的团队协作与沟通能力可助于 最大限度实现个人价值。[7]在她的研究中十分重视人 文与传统文化, 但并未提到设计素养对知识技能的要 求。笔者发现胡文娟、沈榆 (2015) 在讨论设计实践 教育环节的重要性时指出了人文素质与知识技能的 同等重要性, 认为好的设计本身就是一种文化输出, 文化输出就需要符合当地实际情况的人文素养, 故设 计素养是设计技能与人文素质的结合。[8]

阅读以上文献后笔者发现一个共同点: 在设计专 业领域中大家对设计素养的看法总是严谨的。可设计 无处不在, 生活中我们看待设计素养的角度是否会有 所不同呢? 果然, 李盛弘（2019）给了有趣的答案。 他通过分享了生活中几个小故事来讨论设计素养与 设计产品的关系，其中有一句话令人印象深刻：“一 件设计作品的好与坏、成功与失败、实用性与装饰性, 皆可以初略区别在功能上、形态上、造型上、材料上 等等具体的“物体上的改变”, 一件事物所包含的设计
素养应该着重于教育上、心灵上、意识上、体验上等 等较为抽象的“心态上的转变”。 99 零胗几笔, 却突出 了设计素养作为一种软实力有多么重要。

李盛弘对设计素养的思考与国外研究者们较为 相似, 擅长通过一些设计案例或产品找寻设计素养的 内涵。Chris Pacione (2010) 通过与设计师们的谈话 以及莱昂纳多发现了斐波那契数列这个例子指出设 计素养逐渐像数学素养一样重要, 我们需要它来解决 生活、工作、学校中的问题, 设计素养也逐渐成为一 项基本的技能, 不仅在每个人的认知能力的全部范围 内, 且满足于每个人的日常需要。[10]这牵扯出设计素 养一个关键的能力, 即解决问题的能力, 这就要求每 个人具备探究、构思、评估、批判的基本技能及良好 的执行能力, 以解决现实世界问题的复杂性。

Liv Merete Nielsen 、Karen Braenne（2013）则 是在持久耐用的设计产品中发掘设计素养的因子, 与 国内研究者不同的是他们强调产品本身, 所以设计素 养受物质思维与可持续性思维影响。他们认为物质知 识很重要, 需要在目的、使用、生产、运输、生态和 伦理的多元背景下看待物质性。物质性作为设计素养 的一个组成部分, 甚至可能会将设计和物质性的理解 与研究提升到一个更广泛的背景, 影响未来的发展。 其次, 人们也需具备可持续的生态观念, 促进可持续 社会、环境、文化和经济的发展。[3]这意味着设计领 域不仅要追求创新, 环保理念也是我们作为公民的责 任。

\section{表 1 设计领域设计素养分析表}

\begin{tabular}{|c|c|}
\hline 研究者 & 设计领域设计素养维度 \\
\hline 薛拥军 & $\begin{array}{c}\text { 设计能力; 创新能力; 设计职业素养; } \\
\text { 社会责任感 }\end{array}$ \\
\hline 冯䒯 & 社会责任感; 文化底蕴; 团队精神 \\
\hline 胡文娟、沈榆 & 设计知识技能、人文素质 \\
\hline 李盛弘 & 创新企图; 注重设计内涵 \\
\hline Chris Pacione & $\begin{array}{c}\text { 解决问题的能力, 即探究、构思、评 } \\
\text { 估、批判的基本技能; 执行能力; }\end{array}$ \\
\hline Liv Merete & 物质思维; 可持续性思维 \\
Nielsen 、 & \\
Karen Braenne & \\
\hline
\end{tabular}

\section{2. 艺术领域之设计素养因子}

何为艺术? “艺术是通过对环境、现象的捕捉分 析, 由创作者加以个人的情感和体验进行表达的产 物。它是一种能够反映当时地区的社会状态, 创作者 个人情感立场的形式, 从而在一定程度上能够满足人 们的精神需求, 丰富人们的精神生活。总的来说, 艺 术是审美和情感的统一”。[11]溯其源头, 在原始社会 中艺术与设计是不可分割的, 为大众所熟知的拉斯科 洞窟与阿尔塔米拉洞窟岩画正体现了旧石器艺术与 设计的完美结合。原始画家们用线条概括动物的形象 并生动地展示了人类捕猎、养殖的画面, 正是这些画 
面向后世传递了一个信息: 这些壁画不仅是艺术品, 其背后真正的意义在于传授后人生存的经验。到了奴 隶社会, 随着生产力的发展和人类等级的产生, 艺术 的地位逐渐提升, 被冠上“高雅艺术”“文人艺术”等头 衔, 为统治者与贵族所拥有; 设计的地位则不断下降, 由劳动人民所从事，艺术与设计由此走向了分离。后 随着欧洲工业革命的开始, 社会生产力得到极大提 高, 以包豪斯学派为代表的的一批艺术家倡导将生 产、社会与技术相结合, 并且在他们的努力下还原了 艺术的本来面目, 即劳动创造了艺术, 艺术也必然回 归劳动之中，使艺术与设计再一次合体。

艺术与设计在岁月的长河中一直相互促进、相互 影响至今, 与我们的生活密不可分, 这也说明了设计 素养绝不仅仅存在于设计领域里, 艺术领域中也存在 着设计素养的踪迹。

李明 (2013) 认为艺术的创造离不开艺术家丰富 而具创新的艺术感悟，且对于艺术创造而言，“灵感” 是不可或缺的。[12]灵感的产生具有随机性与偶然性, 但其产生的前提却与设计素养有着密切的关联。李明 将创作灵感划分为艺术型灵感与经验型灵感, 艺术型 灵感不仅是灵光一现, 也是设计师意识形态的不同思 维方式或观念的习惯导致智慧的碰发; 而经验型灵感 要求设计师对社会历史与环境充分了解, 并且自身具 备足够的文化修养。与设计一样, 文化底蕴在艺术领 域的设计素养中也是十分重要的影响因子, 这也要热 爱艺术的同时热爱本民族的传统文化, 以此培养其深 厚的艺术设计素养。

同年, 李长亮与罗江玫（2013）强调了设计素养 在当今社会的重要性, 并试图陶艺基础中引入设计素 养的理念, 以“形成设计意识, 提高动手能力”为出发 点, 将二者进行有效融合。[13]研究者指出设计品位在 设计素养中的重要地位, 认为在陶瓷制作过程中需要 具备解读生活中的设计韵味的能力和基本的设计品 位, 且动手能力的培养也是艺术实践中必不可少的一 项能力。本研究虽一直强调如何将设计素养融入与陶 艺基础中, 但对如何融入的方法篇幅过少, 使艺术与 设计之间的联结不够紧密, 无法突出艺术领域中设计 素养的重要作用。

笔者总结该领域设计素养之意涵并制成表 2 。

\section{表 2 艺术领域设计素养分析表}

\begin{tabular}{|c|c|}
\hline 研究者 & 艺术领域设计素养维度 \\
\hline 李明 & 灵感思维; 文化底蕴 \\
\hline 李长亮、罗江玫 & $\begin{array}{c}\text { 设计品位; 动手能力; 独立分析和 } \\
\text { 解决问题的能力 }\end{array}$ \\
\hline
\end{tabular}

\section{3. 教育领域之设计素养因子}

设计素养作为设计教育的关键, 其对设计教育发 展的推动性不言而喻, 在国内外中小学教育里, 无论 是美术学科或是其他学科都应富含生命力从而传递 好的教育。可如何赋予教育生命力呢? 以下研究者们
对教育领域中设计素养意涵的探索或许可以给我们 答案。

随着国内设计教育的不断发展, 关注教育领域中 设计素养的专家学者也不断增多, 故与设计素养相关 的教育研究数量也多了起来。周端云(2014)通过对湖 南某学院 320 名学生进行问卷调查得到学生设计素 养的现状。笔者认为导致现状不佳的原因不仅在于学 生对专业认同度不高, 也与他们学习能力和反思能力 有很大的关系 ${ }^{[14]}$ 。设计素养的培养不只要求学生有设 计意识与思维, 更要求学生要有主动学习和反思能 力, 同时, 兴趣是学习的主要动力, 学生对设计的看 法与态度也是设计教育实施中需要考虑的一个问题。

张晓蒙（2015）是在文献基础上分析设计素养对 几个学科的影响: 如在传统绘画专业中, 以吴冠中作 品《人之家》为例, 画家选取最简单最贴近生活的题 材, 将画面分解为点线面等基本构图元素进行设计重 组, 这体现设计素养的设计能力; 在中小学美术教育 中, 设计素养不仅陶冶学生的情操, 也培养其艺术创 造精神与实践能力, 帮助学生培养广阔的视野及丰富 的想象力、创造力; 在如今的高等教育体系中, 一门 有著巧妙教学结构的课程更能帮助学生摆脱思维定 式的牢笼; 而设计素养中的审美能力对理工科也有著 潜在的联系。[15]该研究看待设计素养的研究与应用较 为全面, 认为设计素养是多维的而非局限于美术教育 中, 且可以为每个学科赋予生命力。

孔紫君（2018）通过归纳法将设计素养的意涵归 纳为以下几个部分: 首先设计素养包含思维能力、表 达能力、感受能力、寻找问题以及独立思考能力; 其 次需要专注的心理素质、意志力; 最后是高尚的道德 素质、科学的文化素质。随后研究者将设计素养与创 新教育联结起来, 分析设计素养在大学生创新教育中 的作用, 如促进学生创新思维的培养、拓展创新教育 的内容以及教育完善健康的人格等。[16]该研究分别解 释了创新教育与设计素养的概念, 层层递进阐明设计 素养与创新人才培养之间的逻辑关系, 从而揭示了设 计素养在学校创新教育中的重要作用, 但由于缺少较 为科学的研究方法, 使作者的理论缺少依据支撑。

李静 (2019) 则是针对中小学美术教育对设计素 养的概念提出个人的见解, 认为设计素养应该是一个 人在生活与学习中所具备的思维创新、带有创造性和 价值判断能力的品质。且这个品质的形成过程是潜移 默化的, 需要长时间的培养与沉淀, 受教育方式、成 长环境等因素影响巨大, 所以应该从小注重对设计素 养的培养。[17]该研究虽与孔紫君的研究一样偏向理论 化, 但“设计素养中具备价值判断能力”这一点尚未有 研究者提起, 令人倍感新奇, 于笔者个人理解而言, 价值判断能力与学生的客观评价能力有关, 需要尊重 个体审美与创造能力的差异性。

国外设计教育起步早, 发展快, 对教育领域中设 计素养的研究更注重实践且相对成熟。Ingvild Digranes、Liv Merete Nielsen（2012）分析挪威教育 
系统中不同教育水平之间的过渡，选择“设计素养”这 一概念以探讨和解决从小学到大学各级设计教育的 目标和内容的复杂问题。[18]研究者通过设计素养的相 关文献分析对设计素养概念进行总结, 认为设计素养 包括对设计产品、设计过程的理解能力, 这不仅是专 业设计师的能力, 也是一般公众的能力; 设计素养也 指关注和实践, 如民主参与设计过程, 发展和使用道 德责任, 以及理解与支持可持续的生产和消费; 设计 素养还体现在对智能的包容性, 且包含决策能力和终 身学习能力。在他们的研究中也很好地体现了西方的 民主思想。

Kasper Skov Christensen、Mikkel Hjorth 等研究者 (2018) 则是通过问卷调查与实验教学等方法评估学 生对探究的态度来理解中学教育中的设计素养。[19] 该研究注重学生的探究能力, 要求学生对探究有明确 的设计立场。其次在教学实践中研究者观察学生解决 棘手问题的能力, 并帮助学生掌握设计过程。最后研 究者提出设计同我们的阅读和写作能力一样是非常 基本的素养, 这意味着学生应该培养基本的设计能 力, 如设计探究、构思、指导设计过程以及将思想外 化的技能。关于思想外化这一点, Eva lutnæs (2020) 在对如何培养负责任的设计素养研究中也曾具体提 起。通过回顾挪威 1-10 年级艺术和手工艺科目的课 程目标, 她认为设计素养中思想外化的能力指学生通 过制造获得意识, 通过熟练的制作和知道如何转换材 料以外化思想, 并知道如何通过使用视觉元素来表达 意义。其次, 在她的研究中也十分注重社会生态责任 和批判性反思能力。[20]

研究教育领域设计素养的专家学者较多, 笔者将 以上研究者与他们对设计素养的定义进行整理, 制成 表3。

表 3 教育领域设计素养分析表

\begin{tabular}{|c|c|}
\hline 研究者 & 设计领域设计素养维度 \\
\hline 周端云 & $\begin{array}{c}\text { 设计能力; 创新能力; 设计职业素养; } \\
\text { 社会责任感 }\end{array}$ \\
\hline 张晓蒙 & $\begin{array}{c}\text { 设计能力; 艺术创想精神; 实践能力; } \\
\text { 审美能力 }\end{array}$ \\
\hline 孔紫君 & $\begin{array}{c}\text { 思维能力、表达能力、感受能力、独立 } \\
\text { 思考能力; 专注的心理素质、意志力; } \\
\text { 道德素质; 文化素质 }\end{array}$ \\
\hline 李静 & 思维创新; 创造性; 价值判断能力 \\
\hline $\begin{array}{c}\text { Ingvild } \\
\text { Digranes、Liv } \\
\text { Merete } \\
\text { Nielsen }\end{array}$ & $\begin{array}{c}\text { 对设计产品、设计过程的理解能力; 民 } \\
\text { 主参与设计过程; 道德责任; 对智能的 } \\
\text { 包容性; 决策能力和终身学习能力 }\end{array}$ \\
\hline $\begin{array}{l}\text { Kasper Skov } \\
\text { Christensen、 } \\
\text { Mikkel } \\
\text { Hjorth 等 }\end{array}$ & $\begin{array}{c}\text { 探究能力: 对探究有明确的设计立场; } \\
\text { 解决棘手问题的能力; 基本的设计能 } \\
\text { 力: 探究、构思、指导设计过程以及将 } \\
\text { 思想外化的技能 }\end{array}$ \\
\hline Eva lutnæs & $\begin{array}{c}\text { 思想外化; 社会生态责任; 批判性反思 } \\
\text { 能力。 }\end{array}$ \\
\hline
\end{tabular}

\section{5.设计素养在中学美术教育中的作用}

与长期以来普通学校设计教育没有受到足够的 重视有关, 我国的基础教育 (小学与中学教育) 阶段 相较于高等设计教育显得更加薄弱。国内中学分为初 中与高中, 两个区段时间跨度不长却构成了人生中最 重要的一个阶段——青春期。美术教育家罗恩菲德教 授曾说过，青春期（13-17 岁）是人生成长中的“黄金 时段”, 这个时段孩子由初步的兴趣爱好、动手能力、 协作精神和设计意识等的形成，到鉴赏评价、创意实 践、理念思辨等能力的逐步提升, 再到最后全面的设 计素养达成, 是一个自然、整体的教育系统。 ${ }^{[21]}$ 上文 提到设计素养之意涵包括设计、艺术、教育三大层面 约 40 项因子, 这些设计素养因子若能结合中学生的 年龄优势, 定能将设计素养对中学美术教育发展的推 动作用发挥至最大化。以下为笔者对设计素养在中学 美术教育中具体作用的归纳与分析。

\section{1. 培养学生的基本能力}

基本能力即中学阶段设计课程对学生的基础能 力要求, 首先是设计基本能力, 包括良好的设计思维 能力、具备解读生活中的设计韵味的能力和基本的设 计品位、设计意识与技能知识; 其次是创新能力, 创 新能力与创新精神一般被认为是基础教育中最核心 的教育目标之一, 创新能力并非是一种单一能力, 它 是由一些思维能力综合而来, 主要包括系统性思维能 力、创造性思维能力与实践能力等; 审美能力也是中 学生美术学科必备的能力与素养; 最后是中学生主动 学习和反思的能力, 青春期的学生由于心理生理的成 长变得比较自我, 很难集中注意力或者对单一事物保 持兴趣, 设计素养的培养可帮助学生调动自身学习主 观能动性、重复学习和反思、以及借鉴他人经验的能 力。

\section{2. 有助于学生关键能力的培养}

关键能力即学生在处理关键问题时应具备的能 力, 如设计素养因子中的独立分析能力、执行能力、 思想外化能力、团队精神、沟通与协作能力以及专注 的心理素质与意志力。青春期的学生认知水平正迅速 发展, 但成人感与半成熟现状之间的矛盾也十分明 显, 这导致学生容易对问题、困难的认知不全面甚至 较为偏执, 故设计素养的培养可在中学生在解决问题 与困难时发挥关键性作用。

\section{3. 发掘学生思维的火花}

我国中学美术教育课程标准中强调教师应该创 造问题情境, 以此激发学生的思维情趣。心理学认为 思维是人对客观事物本质特征和规律性联系大反映, 设计素养对于培养中学生思维品格的作用在于发掘 学生的艺术创新能力、思维创新与创造性, 并且在教 学过程中培养学生逻辑与非逻辑的思维模式, 在课堂 
互动和美术鉴赏课程中, 设计素养有利于培养学生成 长关键期的思辨能力批判性思维。设计素养可激发中 学生的创造力, 帮助学生用辩证的角度看待问题, 也 有利于教师在美术鉴赏课程中培养其成长关键期的 思辨能力与批判精神。

\section{4. 引导学生形成积极的情感态度}

积极的情感态度首先离不开对学生社会责任感 的培养, 这要求学生具备良好的道德修养和伦理意 识。其次, 设计素养培养中学生对本民族传统文化与 艺术的热爱感, 并随着时间的增长使学生培养出深厚 的文化底蕴; 设计素养中的价值判断能力则教会学生 客观评价与判断, 学会尊重个体审美与创造能力的差 异性; 此外, 由于中学生有着强烈的好奇心, 对学生 探究态度的引导也十分利于学生能力的提升和中学 美术教育的发展。

\section{6. 结论}

本文中, 笔者首先分析设计教育的发展并解读设 计素养与设计教育之间的关系, 在这一部分我们了解 到中小学设计教育的实施对设计素养的培养是至关 重要的。然后笔者包围着“设计素养是什么? ”这个问 题分两部分进行解答: (1) 分别叙述设计与素养的 概念; （2）在文献基础上探讨设计素养之意涵，该 部分是以设计、艺术、教育三大领域为目标找寻设计 素养的重要影响因子, 结合以上领域的设计素养因子 最终汇成设计素养的总意涵。最后, 笔者对设计素养 因子在中学美术教育发展中的重要性进行探讨并得 出结论: 设计素养对培养学生的基本能力、关键能力、 思维品质与情感态度有着积极作用。2 1 世纪是人人 参与设计的时代，展望今后设计素养能够如同阅读、 计算一般成为人人必备的基本素养, 且更好地融入到 中学美术教育中, 使教育的生命力代代相传。

\section{REFERENCES}

[1] Xiao Jun, Li Binbin.(2008) Innovative Thinking on Design Art Education for Primary and Secondary S chools.Science.Education and Literature Exchange, 09:93-99.

[2] Liv Merete Nielsen, Karen Braenne. (2013)Design Literacy for Long Lasting Products.Studies in Mate rial Thinking, http://www.materialthinking.org.

[3] Qiang Fensheng. (2000)Comprehensively understan $\mathrm{d}$ the meaningof quality education .China Educatio n Journal(02).doi:CNKI:SUN:ZJYX.2000-02-004.

[4] Zhang Dingqiang. (2019)Instructional Design Litera cy: Connotation Analysis and Development Path. C ontemporary Education and Culture,02:67-71.

[5] Luo Zhufeng. "Chinese Dictionary (1993 Edition)" Chinese Dictionary Publishing House. Shandong.
[6] Xue Yongjun. (2009)On the Improvement of Design Literacy of Art Interior Design Majors . Science an d Technology Information, 17:202-204.

[7] Feng Ying. (2014)Research on Evaluation Criteria o $f$ Design Literacy of Art Design Talents Based on Cultural and Creative Industries. Grand View of Fin e Arts,04:150-151.

[8] Hu Wenjuan, Shen Yu.(2015)The Necessity of Desi gning Practical Education Link-Taking German Ed ucation Mode as an Example .Design, 19:104-105.

[9] Li Shenghong. (2019)The relationship between desi gn literacy and design in life. Design,04:88-89.

[10] Chris Pacione.(2010)Evolution of the Mind: A Cas e for Design Literacy.On Design Thinking. DOI:10 $.1145 / 1699775.1699777$

[11] Zhang Shuying. (2019)Viewing the Reform of Mod ern Art and Design Education from the Relationshi $\mathrm{p}$ between Art and Design. Mass Standardization,1 2:206-208

[12] Li Ming.(2013)On the Cultural Connotation and De sign Accomplishment of Fashion Designers. Popul ar Literature and Art. 10:95-96.

[13] Li Changliang, Luo Jiangmei. (2013)Preliminary St udy on Design Literacy in Basic Education of Cera mic Art in Middle Schools. Art and Design (Theory ), 11:174-176

[14] Zhou Duanyun.(2014)Research on the Accomplish ment Status and Countermeasures of Activity Desi gn of Preschool Education College Students-Takin $\mathrm{g}$ a College in Hunan as an Example. Journal of $\mathrm{H}$ ubei Correspondence University,03:114-115.

[15] Zhang Xiaomeng.(2015)On the Influence of Art De sign Quality Education on Other Disciplines.Journa 1 of Hubei Correspondence University,05:116-117.

[16] Kong Zijun. (2018)On the Role of Design Literacy in Innovative Education of College Students . Art E ducation Research,20:74-75.

[17] Li Jing. (2019)On the Cultivation of Design Literac $y$ in Art Teaching in Primary and Secondary Schoo 1s. Western Leather, 14:146.

[18] Ingvild Digranes,Liv Merete Nielsen.(2012)Design Literacy - From primary education to university 1 evel.Chulalongkorn University.Bangkok, Thailand.

[19] Kasper Skov Christensen.Mikkel Hjorth.(2018)Ole Sejer Iversen.Rachel Charlotte Smith.Understandin $\mathrm{g}$ design literacy in middle-school education: assess ing students\&apos;stances towards inquiry.Int $\mathrm{J} \mathrm{Te}$ chnol Des Educ.https://doi.org/10.1007/s10798-018 $-9459-\mathrm{y}$. 
[20] Eva Lutnæs.(2020)Empowering Responsible Desig n Literacy: Identifying Narratives in a New Curricu lum.RChD: creación y pensamiento,5:11-22.

[21] Xi Weiquan. (2008)Design Education and Its Value in Ordinary Middle Schools.https://kns.cnki.net/K CMS/detail/detail.aspx?dbname $=$ CDFD0911\&filen ame $=2008144530$.nh. 